\title{
Microbial Population and Beneficial Properties of Rhizospheric Soil as Influenced by Different Amendments in Various Land Use Systems: A Review
}

\author{
Neha $^{1 *}$, B. S. Bhople ${ }^{2}$ and Anil Kumar ${ }^{3}$ \\ ${ }^{1}$ Department of Soil Science, Punjab Agricultural University, Ludhiana, Punjab, India \\ ${ }^{2}$ Department of Soil Science, Regional Research Station, Ballowal Saunkhri, Punjab, India \\ ${ }^{3}$ Farm Science Centre, Guru Angad Dev Veterinary \& Animal Sciences University, \\ Tarn Taran, India \\ *Corresponding author
}

Keywords

Land use systems, Microflora,

Rhizosphere, Soil amendments and

Physico-chemical properties

Article Info

Accepted:

12 March 2020

Available Online:

10 April 2020

\begin{abstract}
A B S T R A C T
Plant roots, soil and microbial interactions result in alteration of soil physical and chemical properties that in turn affect the micro-biological properties in the rhizosphere region. The growth and productivity of plant depends upon the diversity and composition of soil microflora present near rhizosphere zone. The aim of this study is to review the effect of organic, inorganic and integrated use of nutrients on soil properties under different land use systems in rhizospheric region. The studies highlight positive as well as negative influence of organic, inorganic and integrated use of fertilizers on the rhizosphere population. As the addition of above material influence physical and chemical properties of soil, they have a direct influence on soil microbial properties. Studies evident deteriorated soil quality as well as health as we follow intensive chemical fertilizers application. Therefore, various researches suggested an improvement in soil health and crop productivity on sustainable basis through conjoint usage of different package of nutrient sources.
\end{abstract}

\section{Introduction}

Rhizosphere is the vital soil microenvironment where the plant roots, soil properties and microbial activity are interconnected. Rhizosphere microorganisms have direct as well as indirect impact on composition and biomass of natural plant populations (Van der Heijden et al., 1998, 2006, 2008; Schnitzer et al., 2011). Therefore, microbial species abundance in rhizosphere can therefore be used as indicator of aboveground plant diversity and productivity. Plant roots, soil and microbial interactions results in alteration of soil physical, chemical properties that in turn affect the 
microbiological properties in the rhizosphere region (Nihorimbere et al., 2011). The beneficial rhizosphere microorganisms can help in maintenance of ecosystem balance through organic matter decomposition and cycling of nutrients that serves as an indicator of land use changes and ecosystem sustainability (Ros et al., 2006; Balser et al., 2010).

The soil physico-chemical properties strongly influence the microbial properties such as (bacteria, fungi and actinomycetes), basal soil respiration, enzymatic activity, microbial biomass carbon, mineralizable carbon, nitrogen, phosphorus, sulphur etc. Such intense microbial properties occur in rhizosphere zone due to presence of several nutrient rich exudates.

Land-use activities specifically related to agricultural practices can have a significant impact on the quantity and activity of soil microbial community and biological health of soil (Das et al., 2011). Joanisse et al., (2007) and Liu et al., (2002) also stated that anthropogenic activities and various soil physico-chemical properties such as soil $\mathrm{pH}$, soil organic matter, texture etc have great influence on soil microbial activity.

Intensive utilization of inorganic fertilizers without organic manures are responsible for deterioration in soil health in terms of soil physical and chemical properties, lowers soil microbial activity as well as soil humus (Anjanappa et al., 2012).

Nambiar (1997) stated that the integrated use of chemical and organic fertilizers is more effective, not only providing greater stability but also maintains a better soil health. The purpose of this study is to review the impact of different soil amendments on rhizosphere microbial communities and soil physicochemical properties under different land use systems.

\section{Soil properties in relation to different soil amendments}

Various soil amendments have greater impact on soil microbiological properties that are also responsible for the maintenance and determination of soil physico-chemical properties such as soil $\mathrm{pH}, \mathrm{EC}$, soil organic matter, nutrient availability in soil that effect crop yield.

Organic mulches are widely used for soil surface application in order to suppress weeds and diseases, control soil temperature and conserve soil moisture conditions (Robinson, 1988; Hoitink and Boehm, 1999).

It has also been recognised that mulches have greater potential to improve soil structure, increase in soil organic matter content and create nutrient cycling patterns more similar to natural ecosystems (Tukey and Schoff, 1963; Roe, 1998).

On the other hand, plant health and soil sustainability could be maintained by liquid organic fertilizers due to availability of soluble nutrients and abundant soil organic matter (Hou et al., 2017 and Dordas et al., 2007). The integration of watering and fertilizer patterns may be attributed to increase in nutrient use efficiency and decrease in nutrient loss risk (Toonsiri et al., 2016 and Ceretta et al., 2010).

Additionally, suitable vermicompost application along with chemical fertilizer could also be result in inhibition of soil pests and soil-borne diseases (Edwards and Norman,2004) and also causes reduction in plant parasitic nematodes and infection rates in plants (Arancon et al., 2002). Brussard et al., (2007) suggested that application of organic amendments is the most effective way of managing biodiversity in the soils (Table $1)$. 
Table.1 The various favourable and unfavourable influences of different soil amendments on soil properties in rhizospheric soil in different land use systems

\begin{tabular}{|c|c|c|c|c|c|c|c|c|c|}
\hline \multirow[t]{2}{*}{ Sr. } & \multirow[t]{2}{*}{$\begin{array}{l}\text { Land use/ } \\
\text { plant } \\
\text { rhizosphere }\end{array}$} & \multirow{2}{*}{\multicolumn{2}{|c|}{ Soil amendment }} & \multicolumn{2}{|c|}{$\begin{array}{c}\text { Physico chemical properties of } \\
\text { soil/ plant characteristics/yield } \\
\text { attributes }\end{array}$} & \multicolumn{2}{|c|}{ Microbial properties/ enzymatic activities } & \multirow[t]{2}{*}{$\begin{array}{l}\text { Place of } \\
\text { study }\end{array}$} & \multirow[t]{2}{*}{ Reference } \\
\hline & & & & Positive & Negative & Positive & Negative & & \\
\hline \multicolumn{10}{|c|}{ Field crops } \\
\hline \multirow[t]{2}{*}{1.} & \multirow[t]{2}{*}{$\begin{array}{l}\text { Rice } \\
\text { rhizosphere }\end{array}$} & Integrated & $\begin{array}{l}50 \% \text { Nitrogen } \\
\text { (recommended) through urea } \\
+ \text { compost/bhattian sludge }\end{array}$ & Nil & Nil & $\begin{array}{l}\text { Maximum count of fungi, } \\
\text { bacteria, diazotroph, PSB, } \\
\text { actinomycetes and } \\
\text { enzymatic activities such } \\
\text { as dehydrogenase, alkaline } \\
\text { phosphatase and urease } \\
\text { activity were also } \\
\text { increased }\end{array}$ & Nil & \multirow[t]{2}{*}{$\begin{array}{l}\text { Punjab, } \\
\text { India }\end{array}$} & \multirow[t]{2}{*}{$\begin{array}{l}\text { Gill et al., } \\
2016\end{array}$} \\
\hline & & Chemical & $\begin{array}{l}\text { 100\% Nitrogen } \\
\text { (recommended)through urea }\end{array}$ & Nil & $\begin{array}{l}\text { Decrease in } \\
\text { soil pH and } \\
\text { increase in } \\
\text { soil EC }\end{array}$ & Nil & $\begin{array}{l}\text { Suppressed } \\
\text { microbial activity }\end{array}$ & & \\
\hline \multirow[t]{2}{*}{2.} & $\begin{array}{l}\text { Wheat } \\
\text { rhizosphere }\end{array}$ & Integrated & $\begin{array}{l}\text { Chemical nitrogen }\left({ }^{15} \mathrm{~N}-\right. \\
\text { labeled } \\
\text { urea }+ \text { swine manure }\end{array}$ & $\begin{array}{l}\text { Nitrogen rate was } \\
\text { two times faster } \\
\text { than inorganic } \\
\text { fertilizer } \\
\text { application. }\end{array}$ & & $\begin{array}{l}\text { Increased microbial } \\
\text { biomass carbon and } \\
\text { increased enzymatic } \\
\text { activities such as } \\
\text { invertase, urease and } \\
\text { protease }\end{array}$ & Nil & \multirow[t]{2}{*}{ China } & \multirow[t]{2}{*}{$\begin{array}{l}\text { Yuan et } \\
\text { al., } 2011\end{array}$} \\
\hline & & Inorganic & $\begin{array}{l}\text { Chemical nitrogen }\left({ }^{15} \mathrm{~N}-\right. \\
\text { labeled } \\
\text { urea) }\end{array}$ & Nil & No changes & Increased urease activity & Nil & & \\
\hline 3. & $\begin{array}{l}\text { Wheat } \\
\text { rhizosphere }\end{array}$ & Organic & $\begin{array}{l}\text { Farm yard manure and } \\
\text { organic liquid booster like } \\
\text { Jeevamruth and Beejamruth }\end{array}$ & Nil & Nil & $\begin{array}{l}\text { Enhances rhizosphere } \\
\text { mycoflora population and } \\
\text { diversity of species- } \\
\text { Acremonium sp., }\end{array}$ & Nil & India & $\begin{array}{l}\text { Shaikh and } \\
\text { Gachand, } \\
2013\end{array}$ \\
\hline
\end{tabular}




\begin{tabular}{|c|c|c|c|c|c|c|c|c|c|}
\hline & & & (Palekar, 2006) & & & $\begin{array}{l}\text { Trichoderma } \\
\text { pseudokonigii, Glomus } \\
\text { sp., Cladosporium } \\
\text { herbarum and Curvularia } \\
\text { lunata (increases soil } \\
\text { fertility), Aspergillus, } \\
\text { Penicillium, Trichoderma, } \\
\text { Fusarium, Rhizopus and } \\
\text { Cladosporium }\end{array}$ & & & \\
\hline & & Inorganic & Chemical fertilizers & Nil & Nil & $\begin{array}{l}\text { Isolated mycoflora } \\
\text { Aspergillus, Penicillium, } \\
\text { Trichoderma, Fusarium, } \\
\text { Rhizopus and } \\
\text { Cladosporium }\end{array}$ & $\begin{array}{l}\text { Lowers the } \\
\text { rhizosphere } \\
\text { mycoflora } \\
\text { population as } \\
\text { compared to } \\
\text { organic }\end{array}$ & & \\
\hline \multirow[t]{2}{*}{4.} & \multirow[t]{2}{*}{ Wheat } & Integrated & $\begin{array}{l}\text { Fertilizers level+FYM+ } \\
\text { bioinoculants (Azotobactor } \\
\text { chroococum, Cd, } \\
\text { Pseudomonas fluorescens } \\
\text { BHU PSB06, acillus } \\
\text { megaterium BHU PSB14) }\end{array}$ & $\begin{array}{l}\text { Increase in water } \\
\text { holding capacity, } \\
\text { organic carbon, } \\
\text { available N, P } \\
\text { and K and } \\
\text { decreased bulk } \\
\text { density }\end{array}$ & Nil & $\begin{array}{l}\text { Increased dehydrogenase, } \\
\text { phosphatase enzyme } \\
\text { activity, soil microbial } \\
\text { biomass carbon and } \\
\text { microbial properties of } \\
\text { soil. }\end{array}$ & Nil & \multirow[t]{2}{*}{ India } & \multirow[t]{2}{*}{$\begin{array}{l}\text { Parewa et } \\
\text { al., } 2014\end{array}$} \\
\hline & & Inorganic & $\begin{array}{l}100 \% \text { NPK (Recommended) } \\
\text { basic fertilizers }\end{array}$ & $\begin{array}{l}\text { Decrease in } \\
\text { nutrient } \\
\text { availability }\end{array}$ & Nil & Nil & $\begin{array}{l}\text { Lower microbial as } \\
\text { well as enzymatic } \\
\text { activity }\end{array}$ & & \\
\hline 5. & Maize & $\begin{array}{l}\text { Different } \\
\text { coated urea }\end{array}$ & $\begin{array}{l}\text { Neem coated urea, } \\
\text { Pongamia oil coated urea } \\
\text { and Castor oil coated urea }\end{array}$ & $\begin{array}{l}\text { Higher NPK } \\
\text { content when } \\
100 \% \text { rec N } \\
\text { applied through } \\
\text { Neem Coated } \\
\text { Urea. }\end{array}$ & $\begin{array}{l}\text { Lower } \\
\text { availability } \\
\text { of nutrients }\end{array}$ & Nil & Nil & India & $\begin{array}{l}\text { Shilpha et } \\
\text { al., } 2017\end{array}$ \\
\hline 6. & Maize & Integrated & $\begin{array}{l}\text { Biochar addition and } \\
\text { nitrogen reduction }\end{array}$ & Nil & Nil & $\begin{array}{l}\text { Influences rhizosphere } \\
\text { metabolome, quality and } \\
\text { quantity of root exudates }\end{array}$ & Nil & China & $\begin{array}{l}\text { Cheng et } \\
\text { al., } 2018\end{array}$ \\
\hline
\end{tabular}




\begin{tabular}{|c|c|c|c|c|c|c|c|c|c|}
\hline & & & & & & $\begin{array}{l}\text { i.e. Increases the levels of } \\
\text { amino acids and organic } \\
\text { acids. }\end{array}$ & & & \\
\hline & & Inorganic & $\begin{array}{l}\text { Nitrogen addition through } \\
\text { urea }\end{array}$ & Nil & Nil & Nil & $\begin{array}{l}\text { Decreases the } \\
\text { rhizosphere } \\
\text { microbial } \\
\text { communities and } \\
\text { quantity and quality } \\
\text { of root exudates } \\
\text { also lesser. }\end{array}$ & & \\
\hline \multirow[t]{2}{*}{7.} & \multirow[t]{2}{*}{ Sugarcane } & \multirow[t]{2}{*}{ Inorganic } & $\begin{array}{l}\text { High dose of nitrogen ( } 200 \\
\mathrm{~kg} \mathrm{~N} / \mathrm{ha} / \text { year) }\end{array}$ & Nil & Nil & Nil & $\begin{array}{l}\text { Ascomycetes fungi } \\
\text { (pathogenic fungi) }\end{array}$ & \multirow[t]{2}{*}{ Australia } & \multirow{2}{*}{$\begin{array}{l}\text { Paungfoo- } \\
\text { Lonhienne } \\
\text { et al., } 2017\end{array}$} \\
\hline & & & $\begin{array}{l}\text { Low dose of nitrogen }(40 \mathrm{~kg} \\
\mathrm{N} / \mathrm{ha} / \text { year })\end{array}$ & Nil & Nil & $\begin{array}{l}\text { Basidiomycetes fungi } \\
\text { (lignin decomposer, helps } \\
\text { in carbon cycling), lesser } \\
\text { abundance of ascomycetes }\end{array}$ & Nil & & \\
\hline \multirow[t]{2}{*}{8.} & $\begin{array}{l}\text { Maize- } \\
\text { cabbage }\end{array}$ & $\begin{array}{l}\text { Bioorganic } \\
\text { fertilizers }\end{array}$ & $\begin{array}{l}\text { Soil amended with organic } \\
\text { fertilizer + Trichoderma } \\
\text { guizhouense NJAU } 4742\end{array}$ & $\begin{array}{l}\text { Higher levels of } \\
\text { soil } \mathrm{pH} \text {, the } \\
\text { concentrations of } \\
\text { total organic } \\
\text { carbon, Total } \mathrm{N} \text {, } \\
\text { total } \mathrm{P} \text {, total } \mathrm{K} \text {, } \\
\mathrm{NH}_{4}-\mathrm{N} \text {, avail } \mathrm{P} \\
\text { and avail } \mathrm{K}\end{array}$ & $\begin{array}{l}\text { Decrease in } \\
\mathrm{NO}_{3}-\mathrm{N}\end{array}$ & $\begin{array}{l}\text { Fungus genera: Humicola, } \\
\text { Derxomyces, } \\
\text { Rhizophydium and } \\
\text { Trichoderma were } \\
\text { significantly higher }\end{array}$ & $\begin{array}{l}\text { Bacterial genera } \\
\text { Zavarzinella, } \\
\text { Rubritepida } \\
\text { And Bdellovibrio, } \\
\text { were significantly } \\
\text { depleted }\end{array}$ & \multirow[t]{2}{*}{$\begin{array}{l}\text { Jiangsu } \\
\text { province, } \\
\text { China }\end{array}$} & \multirow[t]{2}{*}{$\begin{array}{l}\text { Qiao et al., } \\
2019\end{array}$} \\
\hline & & Organic & Chicken manure & -do- & -do- & $\begin{array}{l}\text { Bacterial genus } \\
\text { abundance: } \\
\text { Massilia, Zavarzinella and } \\
\text { Rubritepida Fungus genus } \\
\text { abundance: Massaria, } \\
\text { Naumovozyma, } \\
\text { Cladorrhinum }\end{array}$ & -nil- & & \\
\hline 9. & $\begin{array}{l}\text { Soybean } \\
\text { rhizosphere }\end{array}$ & Organic & $\begin{array}{l}\text { Plant compost }(\mathrm{PC}) \text {, } \\
\text { vermicompost }(\mathrm{VC}) \text {, }\end{array}$ & $\begin{array}{l}\mathrm{Ph}, \text { moisture } \\
\text { content, Total N, }\end{array}$ & $\begin{array}{l}\text { Available } \\
\text { phosphorus }\end{array}$ & $\begin{array}{l}\text { Greater microbial } \\
\text { population of fungi and }\end{array}$ & Nil & India & $\begin{array}{l}\text { Das and } \\
\text { Dkhar, }\end{array}$ \\
\hline
\end{tabular}




\begin{tabular}{|c|c|c|c|c|c|c|c|c|c|}
\hline & & & $\begin{array}{l}\text { Farmyard manure (FYM) } \\
\text { and integrated plant compost } \\
\text { (IPC). }\end{array}$ & exch K (FYM) & (PC). & $\begin{array}{l}\text { bacteria, Soil respiration, } \\
\text { Microbial biomass carbon }\end{array}$ & & & 2011 \\
\hline & & Inorganic & $\begin{array}{l}\text { Nitrogen, phosphorus and } \\
\text { potassium thru' fertilizers }\end{array}$ & $\begin{array}{l}\text { Soil available } \\
\text { phosphorus }\end{array}$ & $\begin{array}{l}\mathrm{pH}, \text { moisture } \\
\text { content }\end{array}$ & Nil & $\begin{array}{l}\text { Lesser bacteria and } \\
\text { fungi population, } \\
\text { Microbial biomass } \\
\text { carbon. }\end{array}$ & & \\
\hline \multirow[t]{2}{*}{10} & \multirow[t]{2}{*}{$\begin{array}{l}\text { Soybean } \\
\text { rhizosphere }\end{array}$} & Organic & $\begin{array}{l}\text { Nitrophospho- } \\
\text { Sulphocompost, } \\
\text { Phosphocompost }\end{array}$ & Nil & Nil & $\begin{array}{l}\text { Significantly higher } \\
\text { enzymatic activities like } \\
\text { urease, DHA, alkaline } \\
\text { Phosphatase, aryl } \\
\text { sulphatase. }\end{array}$ & Nil & \multirow[t]{2}{*}{ India } & \multirow[t]{2}{*}{$\begin{array}{l}\text { Souza et } \\
\text { al., } 2017\end{array}$} \\
\hline & & Inorganic & Urea, MOP, DAP & Nil & Nil & Nil & $\begin{array}{l}\text { Lower enzymatic } \\
\text { activities }\end{array}$ & & \\
\hline \multirow[t]{2}{*}{11} & Chickpea & Integrated & $\begin{array}{l}\text { Inoculation with } \\
\text { Trichoderma koningiopsis } \\
\text { strain (NBRI- } \\
\text { PR5)+FYM+NPK (different } \\
\text { doses) }\end{array}$ & $\begin{array}{l}\text { Enhanced plant } \\
\text { growth } \\
\text { parameters, soil } \\
\text { pH }\end{array}$ & Nil & $\begin{array}{l}\text { Phosphorus solubilization, } \\
\text { modifying the rhizosphere } \\
\text { microbial quantity and } \\
\text { quality as well as } \\
\text { enzymatic activities. }\end{array}$ & Nil & \multirow[t]{2}{*}{ India } & \multirow[t]{2}{*}{$\begin{array}{l}\text { Tandon et } \\
\text { al., } 2018\end{array}$} \\
\hline & & Inorganic & $\begin{array}{l}\text { Commercially available } \\
\text { fertilizers NPK }\end{array}$ & Nil & $\begin{array}{l}\text { Less } \\
\text { beneficial } \\
\text { without } \\
\text { inoculation }\end{array}$ & Nil & $\begin{array}{l}\text { Lesser as compared } \\
\text { to bio-inoculation }\end{array}$ & & \\
\hline \multirow[t]{2}{*}{12} & Red Amaranth & Organic & $\begin{array}{l}\text { Different leaf litter (acacia, } \\
\text { eucalyptus, teak, Sal) }\end{array}$ & $\begin{array}{l}\text { Highest Organic } \\
\text { matter, total } \\
\text { nitrogen, } \\
\text { available } \\
\text { phosphorus, } \\
\text { exchangeable } \\
\text { available calcium } \\
\text { and available } \\
\text { magnesium. }\end{array}$ & Nil & Nil & Nil & \multirow[t]{2}{*}{ Bangladesh } & \multirow[t]{2}{*}{$\begin{array}{l}\text { Sarkar et } \\
\text { al., } 2010\end{array}$} \\
\hline & & Inorganic & Chemical fertilizers & Nil & $\begin{array}{l}\text { The lower } \\
\text { level of }\end{array}$ & Nil & Nil & & \\
\hline
\end{tabular}




\begin{tabular}{|c|c|c|c|c|c|c|c|c|c|}
\hline & & & & & $\begin{array}{l}\text { nutrients } \\
\text { was } \\
\text { observed }\end{array}$ & & & & \\
\hline \multirow[t]{2}{*}{13} & \multirow[t]{2}{*}{$\begin{array}{l}\text { Sitanion } \\
\text { Hystrix and } \\
\text { Agropyron } \\
\text { smithii }\end{array}$} & Fertilized & Chemical fertilizers & Nil & $\begin{array}{l}\text { Decreased } \\
\text { organic } \\
\text { matter and } \\
\text { organic } \\
\text { carbon, }\end{array}$ & Nil & $\begin{array}{l}\text { Decreases in fungal } \\
\text { hyphae length of } \\
\text { rhizosphere of both } \\
\text { grasses, decreased } \\
\text { microbial biomass } \\
\text { (S. Hystrix, ) }\end{array}$ & \multirow[t]{2}{*}{ USA } & \multirow[t]{2}{*}{$\begin{array}{l}\text { Klein \& } \\
\text { Frederick, } \\
1989\end{array}$} \\
\hline & & Control & no treatment & $\begin{array}{l}\text { Higher amount of } \\
\text { soil organic } \\
\text { matter and } \\
\text { organic carbon } \\
\text { were observed }\end{array}$ & Nil & $\begin{array}{l}\text { Increased fungal length, } \\
\text { more microbial biomass. }\end{array}$ & Nil & & \\
\hline \multicolumn{10}{|c|}{ Forest crops } \\
\hline 14 & Poplar & $\begin{array}{l}\text { Integrated } \\
\text { (inorganic }+ \\
\text { biofertilizer } \\
\text { s) }\end{array}$ & $\begin{array}{l}\text { Urea and DAP }(100 \% \text { rec }) \\
\text { fertilizer + } \\
\text { Consortium } \\
\text { biofertilizer/azotobacter/PSB }\end{array}$ & Nil & Nil & $\begin{array}{l}\text { Highest Fungi, bacteria, } \\
\text { diazotroph, PSB, Plant } \\
\text { growth promoting } \\
\text { bacteria, Maximum } \\
\text { enzymatic activities such } \\
\text { as DHA, alkaline } \\
\text { phosphatase and urease } \\
\text { enzyme. }\end{array}$ & Actinomycetes & India & $\begin{array}{l}\text { Khipla et } \\
\text { al., } 2017\end{array}$ \\
\hline \multirow[t]{2}{*}{15} & \multirow[t]{2}{*}{$\begin{array}{l}\text { Eucalyptus } \\
\text { camaldulensis }\end{array}$} & Organics & $\begin{array}{l}\text { Mixture of biofertilizers } \\
\text { (Azotobacter chroococcum, } \\
\text { Bacillus circulans and } \\
\text { Arbuscular mycorrhizal } \\
\text { fungi AMF) }\end{array}$ & $\begin{array}{l}\text { Highest content } \\
\text { of chemical } \\
\text { constituents } \\
\text { (chlorophylls a, b, } \\
\text { carotenoids } \\
\text { content, total } \\
\text { Carbohydrates, N, } \\
\text { P and K \%) }\end{array}$ & - & $\begin{array}{l}\text { Mixture treatment } \\
\text { recorded higher microbial } \\
\text { population, mycorrhizal } \\
\text { colonization (\%) and } \\
\text { Inoculation with mixture } \\
\text { of microorganisms } \\
\text { including } \\
\text { Enzymatic activities, inc } \\
\text { nitrogenase activity. }\end{array}$ & Nil & \multirow[t]{2}{*}{ Egypt } & \multirow[t]{2}{*}{$\begin{array}{l}\text { Kh et al., } \\
2014\end{array}$} \\
\hline & & Control & Without treatments & Nil & $\begin{array}{l}\text { Lesser } \\
\text { content of }\end{array}$ & Nil & Nil & & \\
\hline
\end{tabular}




\begin{tabular}{|c|c|c|c|c|c|c|c|c|c|}
\hline & & & & & $\begin{array}{l}\text { chemical } \\
\text { constituents }\end{array}$ & & & & \\
\hline \multirow[t]{2}{*}{16} & \multirow[t]{2}{*}{$\begin{array}{l}\text { Red oak, } \\
\text { Sugar maple, } \\
\text { Yellow birch. }\end{array}$} & Inorganic & $\begin{array}{l}\text { Fertilized with solid } \\
\text { fertilizer like nitrogen, } \\
\text { phosphorus, potassium, } \\
\text { calcium and magnesium. }\end{array}$ & Nil & Nil & Nil & $\begin{array}{l}\text { Reduction in } \\
\text { carbon dioxide flux } \\
\text { from soil, } \\
\text { suppression of } \\
\text { fungal activity due } \\
\text { to decreased } \\
\text { decomposition rate, } \\
\text { reduction in } \\
\text { microbial } \\
\text { respiration and fine } \\
\text { root biomass } \\
\text { (except no changes } \\
\text { observe in case of } \\
\text { red oak in fine root } \\
\text { biomass) }\end{array}$ & \multirow[t]{2}{*}{ USA } & \multirow[t]{2}{*}{$\begin{array}{l}\text { Phillips } \\
\text { and Fahey, } \\
2008\end{array}$} \\
\hline & & Control & No treatment & Nil & Nil & $\begin{array}{l}\text { More activity of } \\
\text { rhizosphere microbial } \\
\text { activity occurs as compare } \\
\text { to fertilized soil. }\end{array}$ & Nil & & \\
\hline \multirow[t]{2}{*}{17} & \multirow[t]{2}{*}{ Pine forest } & Inorganic & $\begin{array}{l}\text { Fertilized with ammonium } \\
\text { nitrate/urea for } 10 \text { years }\end{array}$ & $\begin{array}{l}\text { Increase in soil } \\
\text { carbon content }\end{array}$ & Nil & Nil & $\begin{array}{l}\text { Reduction in } \\
\text { respiration rate of } \\
\text { microbes, ATP and } \\
\text { microbial biomass } \\
\text { carbon. }\end{array}$ & \multirow[t]{2}{*}{ Sweden } & \multirow[t]{2}{*}{$\begin{array}{l}\text { Arne brant } \\
\text { et al., } 1988\end{array}$} \\
\hline & & Control & No fertilizers added & Nil & $\begin{array}{l}\text { Decrease in } \\
\text { carbon } \\
\text { content }\end{array}$ & $\begin{array}{l}\text { Increased respiration rate, } \\
\text { ATP, Microbial biomass } \\
\text { carbon. }\end{array}$ & Nil & & \\
\hline \multicolumn{10}{|c|}{ Horticultural crops } \\
\hline 18 & Banana & Organic & $\begin{array}{l}\text { Compost prepared from the } \\
\text { mixture of filter mud from } \\
\text { sugar factory, plant residues } \\
\text { and conc. Molasses solution. }\end{array}$ & $\begin{array}{l}\text { Higher } \\
\text { concentrations } \\
\text { Of calcium, } \\
\text { magnesium, }\end{array}$ & Nil & $\begin{array}{l}\text { Enzymes like urease, } \\
\text { catalase, alkaline } \\
\text { Phosphatase, acid } \\
\text { phosphatase and invertase }\end{array}$ & Nil & $\begin{array}{l}\text { Guangxi } \\
\text { province, } \\
\text { South China }\end{array}$ & $\begin{array}{l}\text { Zhang et } \\
\text { al., } 2019\end{array}$ \\
\hline
\end{tabular}




\begin{tabular}{|c|c|c|c|c|c|c|c|c|c|}
\hline & & & & $\begin{array}{l}\text { available } \\
\text { nitrogen, } \\
\text { available } \\
\text { potassium, } \\
\mathrm{Fe}, \mathrm{Zn} \text {, soil } \\
\text { organic carbon } \\
\text { and exchangeable } \\
\text { cation exchange } \\
\text { capacity. }\end{array}$ & & $\begin{array}{l}\text { were significantly higher } \\
\text { than lime }\end{array}$ & & & \\
\hline & & Inorganic & Lime @ 3.1 t ha-1 & Nil & $\begin{array}{l}\text { Significantly } \\
\text { lesser } \\
\text { nutrient } \\
\text { levels than } \\
\text { organic. }\end{array}$ & Nil & $\begin{array}{l}\text { Significantly } \\
\text { decrease in } \\
\text { enzymes, liming } \\
\text { alone was not a } \\
\text { viable approach to } \\
\text { fight against } \\
\text { diseases and acid } \\
\text { soils. }\end{array}$ & & \\
\hline 19 & $\begin{array}{l}\text { Citrus } \\
\text { Grandis var. } \\
\text { Longanyou } \\
\text { rhizosphere }\end{array}$ & $\begin{array}{l}\text { Organic / } \\
\text { Integrated }\end{array}$ & $\begin{array}{l}\text { All applied organic } \\
\text { fertilizers/organic fertilizers } \\
\text { + chemical fertilizers like N } \\
\text { P K }\end{array}$ & $\begin{array}{l}\text { Total } \mathrm{N} \text {, available } \\
\mathrm{N} \text {, available Fe, } \\
\text { available } \mathrm{Mn} \text { and } \\
\text { exchangeable } \mathrm{Mg} \\
\text { and organic } \\
\text { matter was } \\
\text { significantly } \\
\text { higher than } \\
\text { chemical } \\
\text { fertilizers/ } \\
\text { organics were } \\
\text { significantly at } \\
\text { par with } \\
\text { integrated system } \\
\text { but higher than } \\
\text { chemical } \\
\text { fertilizers. }\end{array}$ & Nil & Nil & Nil & China & $\begin{array}{l}\text { Li et al., } \\
2017\end{array}$ \\
\hline
\end{tabular}




\begin{tabular}{|c|c|c|c|c|c|c|c|c|c|}
\hline & & Inorganic & Chemical fertilizers (N P K) & Nil & $\begin{array}{l}\text { Significantly } \\
\text { lower values }\end{array}$ & & & & \\
\hline \multirow[t]{2}{*}{20} & \multirow[t]{2}{*}{$\begin{array}{l}\text { Pomegranate } \\
\text { rhizosphere }\end{array}$} & Organic & $\begin{array}{l}\text { Biofertilizers (A. } \\
\text { Chroococcum + G. } \\
\text { Mosseae) }\end{array}$ & $\begin{array}{l}\text { Maximum uptake } \\
\text { of } \mathrm{N}, \mathrm{P}, \mathrm{K}, \mathrm{Ca} \\
\mathrm{Mg} \text { and } \\
\text { micronutrients. }\end{array}$ & Nil & $\begin{array}{l}\text { Dehydrogenase, alkaline } \\
\text { phosphatase and } \\
\text { nitrogenase, hydrolysis of } \\
\text { fluorescein diacetate in } \\
\text { rhizosphere } \\
\text { Soils }\end{array}$ & Nil & \multirow[t]{2}{*}{ India } & \multirow[t]{2}{*}{$\begin{array}{l}\text { Aseri at } \\
\text { al., } 2008\end{array}$} \\
\hline & & Control & Without any treatments & Nil & Nil & $\begin{array}{l}\text { Infected with native AM } \\
\text { fungi }\end{array}$ & Nil & & \\
\hline \multirow[t]{2}{*}{21} & Guava & Organic & $\begin{array}{l}\text { Biofertilizers (Kotengin, } \\
\text { Biomagic, Hummer, } \\
\text { phosphorine, Rhizobacterin, } \\
\text { Biovit solution }\end{array}$ & $\begin{array}{l}\text { Increased } \\
\text { vegetative growth } \\
\text { measurements } \\
\text { (stem height, } \\
\text { stem diameter, } \\
\text { number of shoots } \\
\text { per plant, number } \\
\text { Of leaves per } \\
\text { plant and leaf } \\
\text { area), leaf } \\
\text { photosynthetic } \\
\text { Pigments content } \\
\text { (chlorophyll A, B } \\
\text { and carotenoids) } \\
\text { were increased as } \\
\text { well as leaf } \\
\text { mineral contents } \\
\text { (N, } \mathrm{P}, \mathrm{K}, \\
\mathrm{Ca}, \mathrm{Mg}, \mathrm{Fe}, \mathrm{Mn} \\
\text { and } \mathrm{Zn} \text { ) }\end{array}$ & \multirow[t]{2}{*}{ Nil } & \multirow[t]{2}{*}{ Nil } & \multirow[t]{2}{*}{ Nil } & \multirow[t]{2}{*}{ Egypt } & \multirow[t]{2}{*}{$\begin{array}{l}\text { Khamis et } \\
\text { al., } 2014\end{array}$} \\
\hline & & Control & $\begin{array}{l}\text { Superphosphate, }\left(\mathrm{NH}_{4}\right)_{2} \mathrm{SO}_{4} \text {, } \\
\mathrm{K}_{2} \mathrm{SO}_{4}\end{array}$ & Nil & & & & & \\
\hline 22 & Tomato & Organic & Chicken manure & $\begin{array}{l}\text { Higher plant } \\
\text { height and higher }\end{array}$ & Nil & Nil & Nil & West Africa & $\begin{array}{l}\text { Agyematn } \\
\text { et al., } 2014\end{array}$ \\
\hline
\end{tabular}




\begin{tabular}{|c|c|c|c|c|c|c|c|c|c|}
\hline & & & & fruit yield & & & & & \\
\hline & & Inorganic & $\begin{array}{l}\text { Unik } 15 \text { + Urea, } \\
\text { Winner + winner, } \\
\text { Winner + Sulfan, (Unik } 15 \text { - } \\
\text { 15:15:15 (N:P: K), Sulphan: } \\
24 \% \text { N, 6\% S (12NO3, } 12 \\
\text { NH4), Winner: 15:9:20 + } \\
\text { 1.8 mgo + 3S + 0.02 Z + } \\
0.15 \text { B + 0.02Mn) }\end{array}$ & $\begin{array}{l}\text { Higher plant } \\
\text { height, highest } \\
\text { fruit yield } \\
\text { (Winner + } \\
\text { Sulfan) than } \\
\text { control }\end{array}$ & Nil & Nil & Nil & & \\
\hline \multirow[t]{2}{*}{23} & \multirow[t]{2}{*}{ Tomato } & Organic & $\begin{array}{l}\text { Vermicompost, compost, } \\
\text { Integrated plant nutrient } \\
\text { system (IPNS). }\end{array}$ & $\begin{array}{l}\text { Improved soil pH } \\
\text { and EC. Highest } \\
\text { number of flower } \\
\text { clusters, fruit } \\
\text { clusters, fruit } \\
\text { yield and plant } \\
\text { height (IPNS) }\end{array}$ & Nil & Nil & Nil & Bangladesh & $\begin{array}{l}\text { Islam et } \\
\text { al., } 2017\end{array}$ \\
\hline & & Inorganic & $\begin{array}{l}\text { Urea, MOP, TSP, borax and } \\
\text { Zn fertilizers }\end{array}$ & Nil & $\begin{array}{l}\text { Comparative } \\
\text { ly lesser } \\
\text { yield } \\
\text { attributes } \\
\text { were } \\
\text { recorded }\end{array}$ & & & & \\
\hline 24 & Cucumber & Integrated & $\begin{array}{l}50 \% \text { (recommended) } \\
\text { through inorganic }+50 \% \\
\text { (recommended) through } \\
\text { poultry manure }\end{array}$ & $\begin{array}{l}\text { Positive effects } \\
\text { on soil } \mathrm{pH} \text {, } \\
\text { electrical } \\
\text { conductivity, } \\
\text { organic carbon } \\
\text { and available } \\
\text { nitrogen, } \\
\text { phosphorus and } \\
\text { potassium. }\end{array}$ & Nil & Nil & Nil & $\begin{array}{l}\text { Maharashtra, } \\
\text { India }\end{array}$ & $\begin{array}{l}\text { Ghayal et } \\
\text { al., } 2017\end{array}$ \\
\hline
\end{tabular}




\begin{tabular}{|c|c|c|c|c|c|c|c|c|c|}
\hline & & Inorganic & Chemical fertilizers & Nil & & & & & \\
\hline \multirow[t]{2}{*}{25} & \multirow[t]{2}{*}{$\begin{array}{l}\text { Cucumber } \\
\text { rhizosphere }\end{array}$} & Organic & $\begin{array}{l}\text { Mulches such as recycled, } \\
\text { groundwood pallets and } \\
\text { composted yard waste }\end{array}$ & $\begin{array}{l}\text { Soil mulched } \\
\text { with compost } \\
\text { yard increases the } \\
\text { CEC, OM, P, K, } \\
\text { Calcium and total } \\
\text { N }\end{array}$ & Nil & $\begin{array}{l}\text { Significantly higher } \\
\text { microbial respiration and } \\
\text { microbial nitrogen, higher } \\
\text { population of fluorescent } \\
\text { Pseudomonas }\end{array}$ & Nil & \multirow[t]{2}{*}{ USA } & \multirow[t]{2}{*}{$\begin{array}{l}\text { Tiquia at } \\
\text { al., } 2002\end{array}$} \\
\hline & & Inorganic & Chemical fertilizers & Nil & Nil & Nil & Nil & & \\
\hline \multirow[t]{2}{*}{26} & $\begin{array}{l}\text { Cucumber } \\
\text { rhizosphere }\end{array}$ & Integrated & $\begin{array}{l}\text { Inorganic compound } \\
\text { fertilizer + Vermicompost }\end{array}$ & $\begin{array}{l}\text { Increase in soil } \\
\text { EC, total } \\
\text { nitrogen, total and } \\
\text { available } \\
\text { phosphorus, } \\
\text { available } \\
\text { potassium and } \\
\text { total carbon } \\
\text { content and } \\
\text { Decrease in soil } \\
\text { pH and bulk } \\
\text { density }\end{array}$ & Nil & $\begin{array}{l}\text { Increased the relative } \\
\text { abundance of beneficial } \\
\text { fungi (Ascomycota, } \\
\text { Chytridiomycota, } \\
\text { Sordariomycetes, } \\
\text { Eurotiomycetes, and } \\
\text { Saccharomycetes) and } \\
\text { decreased those of } \\
\text { pathogenic } \\
\text { fungi (Glomeromycota, } \\
\text { Zygomycota, } \\
\text { Dothideomycetes } \\
\text { Agaricomycetes and } \\
\text { Incertae sedis) }\end{array}$ & Nil & \multirow[t]{2}{*}{ China } & \multirow[t]{2}{*}{$\begin{array}{l}\text { Zhao et al., } \\
2017\end{array}$} \\
\hline & & Inorganic & $\begin{array}{l}\text { Chemical compound } \\
\text { fertilizer }\end{array}$ & Nil & $\begin{array}{l}\text { Lower } \\
\text { availability } \\
\text { of nutrients } \\
\text { and carbon } \\
\text { content }\end{array}$ & Nil & $\begin{array}{l}\text { Lowers the } \\
\text { beneficial fungi and } \\
\text { promote harmful } \\
\text { pathogens. }\end{array}$ & & \\
\hline 27 & $\begin{array}{l}\text { Spinach } \\
\text { rhizosphere }\end{array}$ & Organic & Biochar & $\begin{array}{l}\text { Higher values of } \\
\mathrm{pH}, \text { Eh, total } \\
\text { nitrogen, total }\end{array}$ & Nil & $\begin{array}{l}\text { Higher abundance of } \\
\text { bacteria, fungi and } \\
\text { actinomycetes, }\end{array}$ & Nil & $\begin{array}{l}\text { Liaoning,C } \\
\text { hina }\end{array}$ & $\begin{array}{l}\text { Han et al., } \\
2013\end{array}$ \\
\hline
\end{tabular}




\begin{tabular}{|c|c|c|c|c|c|c|c|c|c|}
\hline & & & & $\begin{array}{l}\text { phosphorus, total } \\
\text { potassium, total } \\
\text { carbon, total } \\
\text { sulphur, } \mathrm{C} / \mathrm{N} \text { ratio } \\
\text { and total carbon } \\
\text { were recorded }\end{array}$ & & $\begin{array}{l}\text { ammonifying bacteria, } \\
\text { azotobacter and } \\
\text { denitrifying bacteria }\end{array}$ & & & \\
\hline & & Control & Without biochar & $\begin{array}{l}\text { Higher total } \\
\text { sulphur, } \mathrm{C} / \mathrm{N} \\
\text { ratio, and total } \\
\text { sodium content }\end{array}$ & Nil & Nil & Nil & & \\
\hline \multirow[t]{2}{*}{28} & \multirow[t]{2}{*}{$\begin{array}{l}\text { Chrysanthemu } \\
\text { m rhizosphere }\end{array}$} & $\begin{array}{l}\text { Liquid } \\
\text { organic } \\
\text { fertilizers }\end{array}$ & $\begin{array}{l}\text { Shrimp extract, plant } \\
\text { decomposition, } \\
\text { vermicompost, seaweed } \\
\text { extracts and fish extracts. }\end{array}$ & $\begin{array}{l}\text { Increase in } \\
\text { nutrient levels } \\
\text { (mineral nitrogen, } \\
\text { available } \\
\text { phosphorus and } \\
\text { potassium) }\end{array}$ & Nil & $\begin{array}{l}\text { Stimulate microbial } \\
\text { activity and functional } \\
\text { diversity }\end{array}$ & Nil & \multirow[t]{2}{*}{$\begin{array}{l}\text { Jiangsu, } \\
\text { China }\end{array}$} & \multirow[t]{2}{*}{ Ji, 2017} \\
\hline & & $\begin{array}{l}\text { Chemical } \\
\text { fertilizers }\end{array}$ & $\begin{array}{l}\text { Nitrogen, phosphorus and } \\
\text { potassium fertilizers }\end{array}$ & Nil & $\begin{array}{l}\text { Decreased } \\
\text { nutrient } \\
\text { levels }\end{array}$ & Nil & & & \\
\hline \multirow[t]{2}{*}{29} & $\begin{array}{l}\text { Arecanut palm } \\
\text { rhizosphere }\end{array}$ & Organic & $\begin{array}{l}\text { Farmyard manure, green } \\
\text { leaf, bone meal and wood } \\
\text { ash }\end{array}$ & $\begin{array}{l}\text { Soil organic } \\
\text { carbon and soil } \\
\text { pH showed } \\
\text { significant results }\end{array}$ & Nil & $\begin{array}{l}\text { Higher microbial } \\
\text { population (bacteria, } \\
\text { fungi, actinomycetes) and } \\
\text { Trichoderma sp. and } \\
\text { Aspergillus sp. were } \\
\text { dominated. }\end{array}$ & Nil & \multirow[t]{2}{*}{$\begin{array}{l}\text { Karnataka, } \\
\text { India }\end{array}$} & \multirow[t]{2}{*}{$\begin{array}{l}\text { Bopaiah } \\
\text { and } \\
\text { Bhat,1981 }\end{array}$} \\
\hline & & Inorganic & $\begin{array}{l}\text { Nitrogen, phosphorus and } \\
\text { potassic fertilizers. }\end{array}$ & Nil & Nil & Nil & $\begin{array}{l}\text { Lesser microbial } \\
\text { population as } \\
\text { compared to } \\
\text { organic }\end{array}$ & & \\
\hline
\end{tabular}


Biochar as one of the organic amendments may affect the microbial biomass in many ways as it provides habitat for microflora, protect against hazards and serves as a substrate for microbes (Thies and Rillig, 2011 and Lehmann and Joseph, 2009). Warnock et al., (2007) observed that addition of biochar resulted in promotion of colonization and abundance of mycorrhizal fungi on plant roots.

The studies highlight that integrated use of different organic and inorganic nutrition package may offer feasible and friendly approach towards soil health maintenance and sustainability. As evident by various studies continuous and sole application of inorganic fertilizers resulted in soil quality deterioration, however, combined use of organic and inorganic sources not only contributes significantly to soil health and productivity, but also increase crop productivity and quality on long term sustainable basis.

\section{Acknowledgement}

The authors are highly thankful to researchers whose findings are included directly or indirectly in preparing this manuscript.

\section{References}

Agyeman, K., Osei-Bonsu, I., Berchie, J.N., Osei, M.K., Mochiah, M.B., Lamptey, J.N., Osei, K., and Bolfrey-Arku,K., 2014. Effect of poultry manure and different combinations of inorganic fertilizers on growth and yield of four tomato varieties in Ghana. Agri. Sci. 4, 27-34.

Anjanappa, M., Venkatesh, J., Suresh, and Kumara, B., 2012. Influence of organic, inorganic and biofertilizers on flowering, yield and yield attributes of cucumber (cv. Hassan Local) in open field condition. J. Agri. Sci. 25, 493497.

Arancon, N.Q., Edwards, C.A., and Bierman, P., 2006. Influences of vermicomposts on field strawberries: part 2. Effects on soil microbiological and chemical properties. Bioresour. Technol. 97, 831840.

Arnebrant, K., and Soderstrom, B., 1992. Effects of different fertilizer treatments on ectomycorrhizal colonization potential in two scots pine forests in Sweden. For. Ecol. Manage. 53, 77-89.

Aseri, G.K., Jain, N., Panwar, J., Rao, A.V., Meghwal, P.R., 2008. Biofertilizers improve plant growth, fruit yield, nutrition, metabolism and rhizosphere enzyme activities of pomegranate (Punica granatum L.) in Indian Thar desert. Scientia Horticulturae 117, 130135.

Balser, T.C., Wixon, D., Moritz., 2010. The microbiology of natural soils. In: Dixon G R abd Tilston E L (Eds.) Soil Microbiology and Sustainable Crop Production. pp. 27-58. Springer, Heidelberg.

Bopaiah, B.M., and Bhat N.T., 1981. Effect of continuous application of manures and fertilizers on rhizosphere microflora in arecanut palm. Plant Soil 63, 497-499.

Brussaard, L.D., Ruiter, P.C., and Brown, G.G., 2007. Soil biodiversity for agricultural sustainability. Agri. Eco. Environ. 121, 233-244.

Ceretta, CA., Girotto, E., Lourenzi, C.R., Trentin, G., Vieira, RCB., and Brunetto, G., 2010. Nutrient transfer by runoff under no tillage in a soil treated with successive applications of pig slurry. Agric. Ecosyst. Environ. 139, 689-699.

Cheng, N., Peng, Y., Kong, Y., Li, J., and Sun, C., 2017. Combined effects of biochar addition and nitrogen fertilizer reduction on the rhizosphere metabolomics of maize (Zea mays L.) 
seedlings. Plant Soil. 433, 1-17.

Das, B.D., and Dkhar, M.S., 2011. Rhizosphere microbial populations and physico chemical properties as affected by organic and inorganic farming practices. American-Eurasian J. Agric. Environ. Sci. 10, 140-150.

Dordas, C.A., Lithourgidis, A.S., Matsi, T., Barbayiannis, N., 2007. Application of liquid cattle manure and inorganic fertilizers affect dry matter, nitrogen accumulation, and partitioning in maize. Nutr. Cycl. Agroecosyst. 80, 283-296.

Edwards, C.A., and Norman, Q.A., 2004. Vermicomposts suppress plant pest and disease attacks. Pro. Quest. Agric. J. 45, 51-54.

Ghayal, R.G., Vaidya, K.P., and Tapkeer, P.B., 2017. Effect of different organic manures and inorganic fertilizers on chemical properties of cucumber (Cucumis sativus L.) in lateritic soils of Konkan. Int. J. Chem. Stud. 5: 16261630.

Gill, G.K., Gosal, S.K., and Sharma, S., 2016. Microbial activities and soil health in rice rhizosphere as affected by long term integrated use of organic and inorganic fertilizers. Int. J. Curr. Microbiol. App. Sci. 5: 568-580.

Han, G., Meng, J., Zhang, W., and Chen, W., 2013. Effect of biochar on microorganism's quantity and soil physicochemical property in rhizosphere of spinach (Spinacia oleracea L.). Appl. Mech. Mat. 298: 210-219.

Hoitink, H.A.J., Boehm, M.J., 1999. Biocontrol within the context of soil microbial communities: a substratedependent phenomenon. Аnnu. Rev. Phytopathol. 37, 427-446.

Hou, J.Q., Li, M.X., Mao, X.H., Hao, Y., Ding, J., Liu, D.M., Xi, B.D., and Liu, H.L., 2017. Response of microbial community of organic-matter- impoverished arable soil to long-term application of soil conditioner derived from dynamic rapid fermentation of food waste. Plos one 12, 45-52.

Islam, M.A., Islam, S., Akter, A., Rahman, M.H., and Nandwani, D., 2017. Effect of organic and inorganic fertilizers on soil properties and the growth, yield and quality of tomato in Bangladesh. Agriculture 7, 18-25.

Ji, R., Dong, G., Shi, W., Min, J., 2017. Effects of liquid organic fertilizers on plant growth and rhizosphere soil characteristics of chrysanthemum. Sustainability. 9, 841-849

Joanisse, G.D., Bradley, R.L., Preston, C.M., and Munson, A.D., 2007. Soil enzyme inhibition by condensed litter tannins may drive ecosystem structure and processes: the case of Kalmia angustifolia. New. Phytol. 175, 535546.

Kh, Z., Al-Hadad., Soliman, A.S, Morsy, E.M., Kamel, S.M., El-Sayed, A.A., 2014. Effect of different biofertilizers and soil media on growth and chemical composition of Eucalyptus camaldulensis in North Africa. J. Horti. Sci. Ornament. Plants. 6, 59-70.

Khamis, M.A., Sharaf, M.M., Bakry, K.H., and Abdel- Moty, A.S., 2014. Response of guava transplants to somebiofertilizers. Mid. East. J. Agri. Res. 3, $1184-1188$.

Khipla, N., Gosal, S.K., Gill, and R.I., 2017.Influence of biofertilizers and inorganic fertilizers on soil microbial population and enzyme activities in rhizosphere of poplar. Chem. Sci. Rev. Lett. 6, 2324-2331.

Klein, D.A., and Frederick, B.A., 1989. Fertilizer effects on soil microbial communities and organic matter in the rhizosphere of Sitanion hystrix and Agropyron smithii. Arid Soil Res. Rehabilit. 3, 397-404. 
Lehmann, J., and Joseph, S., 2009. Biochar for Environmental Management (Eds.), London.

Li, R., Chang, Y., Hu, T., Jiang, X., Liang, G., Lu, Z., Y, Y., and Guo, Q., 2017. Effects of different fertilization treatments on soil, leaf nutrient and fruit quality of Citrus grandis var. longanyou. World J. Engineer Technol. $5,1-14$.

Liu, Z.G., Zou, X.M., 2002. Exotic earthworms accelerate plant litter decomposition in a Puerto Rican pasture and a wet forest. Ecol. Appl. 12, 14061417.

Nambiar, M.K.K., 1997. Soil health and organic matter: Changing scenario. Proc. Nat. Acad. Sci. India. 141-160.

Nihorimbere, V., Ongena, M., Smargiassi, and M., Thonart, P., 2011. Beneficial effect of the rhizosphere microbial community for plant growth and health. Biotechnol. Agron. Soc. Environ. 15, 327-337.

Palekar, S., 2006. Text book on shoonya bandovalada naisargika krushi, published by Swamy Anand, Agri Prakashana, Bangalore.

Parewa, H.P., Yadav, J., and Rakshit, A., 2014. Effect of fertilizer levels, FYM and bioinoculants on soil properties in inceptisol of Varanasi, Uttar Pradesh, India. Int J Agri Environ Biotechnol 7, $517-525$.

Paungfoo-Lonhienne, C., Wang, W., Yeoh, Y.K., and Halpin, N., 2017. Legume crop rotation suppressed nitrifying microbial community in a sugarcane cropping soil. Nature 7, 16707.

Phillips, R.P., and Fahey, T.J., 2008. The influence of soil fertility on rhizosphere effects in northern hardwood forest soils. Soil Sci. Soc. Am. J. 72, 453-61.

Qiao, C., Ryan, Penton, C., Xiong, W., Liu, C., Wang, R., Liu, Z., Xu, Xu., Li, R., and Shen, Q., 2019. Reshaping the rhizosphere microbiome by bio-organic amendment to enhance crop yield in a maize-cabbage rotation system. Appl. Soil. Ecol. 142, 136-146.

Robinson, D.W. 1988. Mulches and herbicides in ornamental plantings. Hort. Sci. 23, 547-552.

Roe, N.E., 1998. Compost utilization for vegetable and fruit crops. Hort. Sci. 33: 934-937.

Ros, M., Klammer, S., Knapp, B., Aichberger, K., and Insam, H., 2006. Long term effects of compost amendment of soil on functional and structural diversity and microbial activity. Soil Use. Manag. 22, 209-218.

Sarkar, U.K., Saha, B.K., Goswami, C., and Chowdhury, M.A.H., 2010. Leaf litter amendment in forest soil and their effect on the yield quality of red amaranth. $J$. Bangladesh Agril. Univ. 8, 221-226.

Schnitzer, S.A., Klironomos, J.N., HilleRis, and Lambers, J., et al., (2011) Soil microbes drive the classic plant diversity-productivity pattern. Ecology 92, 296-303.

Shaikh, N.F., and Gachande, B.D., 2013. Effect of organic bio-booster and inorganic inputs on rhizosphere mycoflora population and species diversity of wheat. Int. J. Sci. Res. 4, Article ID: SUB158726

Shilpha, S.M., Soumya, T.M., Girijesh, G.K., and Dhananjaya, B.C., 2017. Effect of different natural oil coated urea fertilizers on productivity and nutrient uptake of maize. J. Pure. App. Biosci. 5, 807-812.

Souza, A.D., Deshmukh, P.W., and Bhoyar, S.M., 2017. Effect of enriched composts on rhizosphere so enzymatic activity of soybean in vertisols. Int. J. Curr. Microbiol. App. Sci. 6, 105-111.

Tandon, A., Fatima, T., Gautam, A., Yadav, U., Srivastava, S., and Singh, P.C., 2018. Effect of Trichoderma 
koningiopsis on chickpea rhizosphere activities under different fertilization regimes. J. Soil. Sci. 8: 261-275.

Thies, J.E., and Rillig, M.C., 2011. Characteristics of biochar: biological properties, pp 85-105.

Tiquia, S.M., Lloyd, J., Hermsb, D.A., Hoitink, H.A.J., Frederick, C., and Jr, M., 2002. Effects of mulching and fertilization on soil nutrients, microbial activity and rhizosphere bacterial community structure determined by analysis of TRFLPs of PCR-amplified 16S rRNA genes. Appl. Soil Ecol. 21, 31-48.

Toonsiri, P., Del, Grosso, SJ., Sukor, A., and Davis, J.G., 2016. Greenhouse gas emissions from solid and liquidorganic fertilizers applied to lettuce. J. Environ. Qual. 45, 1812-1821.

Tukey, R.B., and Schoff, E.L.0., 1963. Influence of different mulching materials upon the soil environment. Proc. Am. Soc. Hort. Sci. 82, 68-76.

Van, der., Heijden, MGA., Bakker, R., Verwaal, J., Scheublin, T.R., Rutten, M., Van, Logtestijn, R., and Staehelin, C., 2006. Symbiotic bacteria as a determinant of plant community structure and plant productivity in dune grassland. FEMS. Microbiol Ecol. 56, 178-187.

Van, der., Heijden, MGA., Bardgett, R.D., and Van, Straalen, NM., 2008. The unseen majority: soil microbes as drivers of plant diversity and productivity in terrestrial ecosystems. Ecol. Lett. 11, 296-310.

Van, der., Heijden, MGA., Boller, T., Wiemken, A., and Sanders, IR., 1998. Different arbuscular mycorrhizal fungal species are potential determinants of plant community structure. Ecology 79, 2082-2091.

Warnock, D.D., Lehmann, J., Kuyper, T.W., and Rillig, M.C., 2007. Mycorrhizal responses to biochar in soil concepts and mechanisms. Plant Soil 300, 9-20.

Yuan, L., Bao, D.J., Jin, Y., Yang, Y.H., and Huang, J.G., 2011. Influence of fertilizers on nitrogen mineralization and utilization in the rhizosphere of wheat. Plant Soil 343, 187-193.

Zhang, J., Bei, S., Li, B., Zhang, J., Christie, P., and Li, X., 2019. Organic fertilizer, but not heavy liming, enhances banana biomass, increases soil organic carbon and modifies soil microbiota. App. Soil. Ecol. 136, 67-79.

Zhao, HT., Li, TP., Zhang, Y., Hu, J., Bai, YC., Shan, YH., and Ke, F., 2017. Effects of vermicompost amendment as a basal fertilizer on soil properties and cucumber yield and quality under continuous cropping conditions in a greenhouse. J. Soil. Sediment. 17, $2718-2730$.

\section{How to cite this article:}

Neha, B. S. Bhople and Anil Kumar. 2020. Microbial Population and Beneficial Properties of Rhizospheric Soil as Influenced by Different Amendments in Various Land Use Systems: A Review. Int.J.Curr.Microbiol.App.Sci. 9(04): 1584-1600. doi: https://doi.org/10.20546/ijcmas.2020.904.186 